\title{
Antecedentes y Consideraciones para la Renovación Curricular de la Carrera de Odontología de la Universidad de La Frontera, Chile
}

\author{
Background and Considerations for the Curricular Renewal of the \\ Universidad de La Frontera, Chile Dental Degree
}

Ramón Fuentes Fernández ${ }^{1} \&$ Claudia Riquelme Macalusso²

FUENTES, F. R. \& RIQUELME, M. C. Antecedentes y consideraciones para la renovación curricular de la carrera de Odontología de la Universidad de La Frontera, Chile. Int. J. Odontostomat., 15(1):234-239, 2021.

RESUMEN: El presente artículo tiene por objeto aportar a la discusión y reflexión de los elementos teóricos-conceptuales que debe incorporar un proceso de renovación curricular, en este caso de la carrera de odontología de la Universidad de La Frontera, Chile. Este proceso debe orientarse hacia los nuevos contextos laborales que requiere hoy en día la odontología; estableciendo diseños y lineamientos claros para el desarrollo de los aprendizajes significativosde los estudiantes -con herramientas específicas, saberes interdisciplinarios y competenciastécnicas para la formación integral del profesional-y que a su vez logre incorporar en todas sus etapas no tan a los estudiantes sino que también a su cuerpo docente. A fin que se logre una apropiación significativa entre todos los actores que participan, adquiriendo un compromiso y alineación con este desafío educativo-formativo, cuyo propósito es la mejora sustancial en la formación profesional odontológica en la región de LaAraucanía, Chile.

PALABRAS CLAVE: renovación curricular, proceso enseñanza-aprendizaje, competencias profesionales.

\section{INTRODUCCIÓN}

Para Díaz-Barriga (2010) desde finales de los años 90's y comienzo del siglo XXI las universidades han tenido que desarrollar las capacidades internas para la generación de trasformación de los programas de pregrado como el posgrado; es por ello que de acuerdo a lo planteado por Pey \& Chauriye (2011) existe hoy en día:

1. La gran acumulación de conocimiento y la velocidad de generación de éste, hacen imposible transmitirlo de la manera tradicional ni puede pretenderse que en los años de duración de las carreras se abarque su totalidad.

2. El convencimiento que las personas formadas se verán enfrentadas durante su vida laboral a un escenario cambiante, demanda una estructura formativa de mayor flexibilidad y con posibilidades de continuidad de estudios.
3. El aumento de cobertura de la educación terciaria o superior, implica un mayor número no sólo de estudiantes, sino que también de académicos docentes, lo que hace que las instituciones deban buscar estrategias que garanticen calidad y pertinencia formativa.

En este contexto, el proceso de renovación curricular, es un mecanismo de aseguramiento de la calidad formativa del profesional, ya que no tan solo responde las necesidades del medio para la actualización de las competencias técnica-profesionales propias de ejercicio laboral, sino que más bien permite que la carrera proyecta su continuidad y sello formativo; y para el logro de ello, se requiere tener presente tres ejes centrales:

- Primero: la necesidad de trabajar una formación centrada en el estudiante, eso es entregando oportuni-

${ }^{1}$ Dental School, Research Centre for Dental Sciences (CICO), Universidad de La Frontera, Temuco, Chile.

${ }^{2}$ Dental School, Universidad de La Frontera, Temuco, Chile.

Received : 24-03-2020 Accepted : 23-09-2020 
dades ciertas de aprendizaje significativo a cada uno de ellos, con metas y resultados claramente establecidos (Fuentes et al., 1996).

- Segundo: No olvidando el desarrollo de competencias genéricas como herramienta indispensable en el mundo actual para enfrentar con acierto los cambios y desafíos de una sociedad que demanda cada vez más la formación integral del ser humano.; y

- Tercero: la formación de un profesional socialmente responsable que dé cuenta de su labor desde una mirada autocrítica y responsable consigo mismo, los demás y el entorno (Vicerrectoría de Pregrado, 2008).

Sin embargo, hay que tener algunas consideraciones para llevar cabo la instalación de un proceso de renovación curricular, ya que se debe siempre detectar las demandas en distintos aspectos, no solo los académicos, ya que la implementación de este proyecto macro hace necesario la existencia de un plan progresivo complejo y de alto costo para una carrera. Para Améstica-Rivas et al. (2017) la:

1. Renovación curricular es un hecho complejo para cualquier universidad, y el avanzar más rápidamente depende no sólo de disponer de un buen proyecto alineado con su misión institucional, sino de disponer de los recursos necesarios para su implementación en la cantidad y plazos necesarios, para asegurar la sustentabilidad académica y financiera de la institución.

2. Evaluación económica de una carrera de pregrado puede diferir una de otra, dada sus propias especificidades, por lo tanto, es imposible inferir que el costo obtenido para la renovación curricular de una carrera es transferible a otra en su totalidad, siendo necesario evaluarse independientemente.

3. Financiación eficiente es uno de los objetivos centrales que se pretende alcanzar en el sistema universitario, haciéndose imperativa la financiación de los modelos educativos que aseguren el éxito y, a su vez minimicen las necesidades económicas, en un contexto de racionalización de la gestión económica de las universidades.

Finalmente, como elemento central en este proceso, es la perspectiva de los docentes y académicos que participan en ella, ya que estos temas referentes a innovaciones curriculares suelen ser siempre creativos desde el inicio, con un alto grado de desconocimiento de los sus fundamentos en las teorías del aprendizaje y desarrollo en que se sustentan; lo que se traduce en problemáticas que deben enfrentan los académicos y docentes son producto de la carencia de protocolos o políticas que faciliten los procesos curriculares de modificación de los programas (DíazBarriga). En ese sentido será importante considerar que la renovación curricular no es tan solo un nuevo plan de estudios, sino que más bien será el producto que implica cambios relevantes, y como plantean Pey \& Chauriye, tiene relación en cuanto a la:

1. Estructura curricular: en la mayoría de los casos se decide continuar con la estructura de asignaturas, identificando en cada una de ellas cuáles son las competencias nuevas que se desarrollarán.

2. Metodología: se reconoce la necesidad de adecuar las metodologías de enseñanza, introduciendo elementos más participativos y de fomento del auto aprendizaje.

3. Evaluación: un aspecto metodológico que requiere especial atención es la evaluación de los logros de aprendizaje.

4. Capacitación docente: se han vinculado a la formación basada en competencias, la capacitación de los docentes que deben innovar en materias de metodología y evaluación, y por ello deben considerar el tiempo de dedicación del estudiante, así como realizar tareas orientadas al cumplimiento del Perfil de Egreso.

5. Sustentabilidad: las transformaciones que implica los cambios curriculares deben ser introducidas cuidando la sustentabilidad económica del programa para evitar encarecer el proceso, eso significa que la toma de decisiones en cuanto este tema dependerá de la viabilidad económica que la institución asegure para el logro del propósito de la renovación curricular.

Un tema central en todo proceso de renovación curricular es la participación activa de los estudiantes, así como también la de los docentes y académicos que participan en el programa. Serán ellos además quienes tengan que requerir una formación especialidad en la temática, que toda renovación curricular debe incorporar y considerar cuando se inicia este tipo de proceso (Duarte \& Mora, 2016). Sin embargo, es importante destacar que cualquiera que sea el modelo 
curricular que se opte, éste debe considerar un sistema evaluación de las competencias del proceso de enseñanza-aprendizaje de los estudiantes a través del logro de los saberes y técnica para tu egreso. Ya que la formación odontológica está centrada en la capacidad del saber hacer -pero integrando la conjunción de los saberes complejos e integrales- lo que permite en el futuro profesional capacidades y competencias para enfrentar los diversos contextos de cambios a través de la crítica y autocrítica de sus propios saberes y aprendizaje formativos en odontología (Ríos Muñoz \& Herrera Araya, 2017).

Entonces¿Qué consideraciones son necesarias para un proceso de renovación curricular de la carrera de Odontología de la UFRO?. El escenario odontológico para el desarrollo de una innovación curricular es distinto a las demás carreras de la salud o de las carreras profesionales en general, debido a la diversidad de complejidades que implica la incorporación de competencias y técnicas en el proceso formativo de los odontólogos hace necesario adecuaciones específicas para la implementación de un proyecto de renovación curricular para que se logre los resultados esperados. Conocer la situación de base de la formación específica que sea descrito permite que se puedan introducir los cambios para una gestión diferenciada (Ruay et al., 2016), ya que el nuevo currículo debe responder no tan solo a las necesidades del contexto donde se desenvuelve, sino que tiene que velar en la adquisición de capacidades para el logro del Perfil de Egreso. Para Duarte y Mora, es importante que existan los siguientes tres ejes para el logro de esto propósitos, siendo importante tener en cuenta:

- La estrecha vinculación entre teoría y práctica.

- El mejoramiento y monitoreo constante de las prácticas.

- El desarrollo de competencias cognitivas que favorezcan el pensamiento crítico.

Asimismo, la renovación curricular además de responder a los requerimientos disciplinarios en coherencia con las necesidades de la salud oral debe considerar también las problemáticas sociales y epidemiológica de la comunidad donde se desarrolla la carrera (Daza, 2010) y los cirujanos dentistas titulados irán a trabajar. Así también, la formación académica del cirujano dentista debe: i) actualizar permanentemente sus conocimientos técnicos y científicos; y ii) aplicar todos los conocimientos técnico-científicos que haya adquirido. Debe considerar también el velar el cumplimiento de su rol en las políticas públicas de salud en los distintos espacios públicos y privados que un cirujano dentista general desarrolla en su ejercicio profesional; considerando que la especialidad constituye otro ámbito de acción que en una renovación curricular en pregrado no tiene una mayor injerencia, pues el foco está en el campo laboral del cirujano general y no en de especialidad. Por ende un plan de estudios de formación general de un odontólogo debe:

- Desarrollar competencias y métodos de aprendizaje orientados hacia una mayor empleabilidad a través de prácticas externas para enriquecer la formación de los estudiantes y tener un conocimiento de las competencias que necesitaran para tener éxito dentro del mercado laboral

- Integrar las ciencias odontológicas con ciencias básicas, humanidades, ciencias sociales, y ciencias de la conducta tomando como centro las necesidades del estudiante para estimular el auto aprendizaje, promoviendo la investigación y apoyar su capacidad para resolver problemas (Miranda, 2013).

Ante lo expuesto, el proyecto académico original de la carrera de Odontología de la Universidad de La Frontera, se caracterizó por poseer una malla curricular, con énfasis en la formación clínica orientada a formar profesionales preparados para enfrentar la salud oral del individuo y de la comunidad, de este modo se consideró un desarrollo que permite una correlación adecuada entre la práctica profesional y las necesidades bucodentales de la población, enfatizando en su formación la consideración de los aspectos psicosociales y biológicos en la resolución de problemas de salud individuales de manera directa con las personas y con un acercamiento temprano en los servicios asistenciales, con un alto compromiso con la promoción y prevención de la salud, de manera de favorecer la formación de un profesional integral del odontólogo. Pedagógicamente se orienta a la combinación de la enseñanza tradicional con una metodología educativa innovada; dando énfasis en el aprendizaje basado en problemas, estimulando el aprendizaje y desarrollo autónomo y la autoevaluación de los estudiantes con un enfoque centrado en ellos mismos, enfatizando su propio rol en el proceso de enseñanza aprendizaje y apoyar sus potencialidades profesionales -este modelo resalta al estudiante como un agente activo en la educación, que a la vez propone y critica su proceso de aprendizaje- así la formación clínica se desarrolla con 
un enfoque integral para que los estudiantes desarrollen las habilidades en forma incremental desde actividades pre-clínicas, y acciones de menor a mayor complejidad clínica, de modo que al culminar sus estudios hayan desarrollado competencias vinculadas al desempeño profesional con enfoque de riesgo y con competencias genéricas que son sello de la Universidad (Matamala et al., 1992; Pantoja et al., 1993; Fuentes et al., 1993, 1996). Es así como la Carrera de Odontología se caracterizan por:

- Que nuestros cirujanos dentistas adquieran las mismas competencia que los titulado de odontología de otra carrera en el país, sobre todo en cuanto a las competencias clínicas específicas que es trasversal a toda formación odontológica.

- La educación en grupos pequeños en base a Aprendizaje Basado en Problemas (ABP) que se trabaja en la línea curricular de Unidad de Integración.

- Una formación con una fuerte orientación en prevención en salud oral, que se potencia en la línea curricular de Salud Pública.

- Formación preclínica odontológica sin fantomas, con acciones incrementales en complejidad, para desarrollar un concepto de cuidado de la persona más que de la afección, integrando el sistema estomatognático al individuo y su contexto de forma holística.

- Clínicas Integradas con enfoque de manejo de factores de riesgo y visión biopsicosocial.

- Participación en los Servicios de Salud Públicos, principalmente atención primaria y enfoque preventivo, durante los dos semestres de Prácticas Profesionales Controladas.

- Fuerte y consolidada formación clínica intramuros.

Desde el inicio de la carrera, el plan de estudios tuvo varios ajustes y modificaciones a través del tiempo, sin embargo, no ha realizado un proceso de renovación curricular completo desde el año 1992, ya que tan solo se ha ido renovando de acuerdo a las necesidades y requerimientos del entorno, debido a que la solidez del perfil creado ha permitido llevar a cabo los propósitos formativos descritos anteriormente. Para llevar a cabo este proceso, requiere nuevamente un análisis curricular profundo del perfil y objetivos formativos para el profesional de la odontología para los próximos 25 años, ya que sean actualizaciones e innovaciones del Plan de Estudios relacionadas con el diseño curricular o actualización de programas de asignatura e incorporación de competencias genéricas en el currículo se requiere de:
1. Un diseño curricular que inicie con el levantamiento de información de los titulados de los últimos 5 años, empleadores de todos los sectores (públicos y privados) y los mismos estudiantes que están cursando la carrera, lo que hace que se pueda construir un perfil del titulado pertinente y consistente renovado;

2. Tener en consideración el perfil del estudiante que están cursando la carrera en cuanto a sus capacidades, conocimientos adquiridos en la enseñanza media y carencias tanto formativas como personales, como por ejemplo si bien es cierto que la mayoría son nativos digitales, no necesariamente utilizan las herramientas del uso de las TIC's para su formación.

3. Una actualización de Programas que permitiría mantener vigentes y acordes los lineamientos formativos las diversas actividades curriculares, respecto a los resultados de aprendizaje demostrables por el estudiante, contenidos temáticos, estrategias y métodos de aprendizaje y de evaluación, establecidas en el programa de asignatura;

4. Competencias Genéricas en el Currículo que entregarían los lineamientos a seguir para la incorporación de competencias genéricas en el currículo durante todo el período de formación para el logro del perfil del titulado (Vicerrectoría de Pregrado, 2018).

Un elemento central en este proceso que no se debe perder de vista es que la mayoría de los programas que se dan la tarea de hacer una renovación curricular siempre se orientan a definir un modelo educativo propio, es por ello que se debe resguardar el desarrollo del currículo que ayuda a entender la problemática que enfrentan los programas y quienes deben participar en ellos, debido a que ante la diversidad de modelos y propuestas curriculares que existen hoy en día, ha sido bajo el campo investigativo sobre significados y cómo han sido llevados a la práctica y sus resultados de la infinidad de casos de programas renovados curricularmente.

Sin perjuicio de lo anterior, lo que se ha mencionado corresponden a los aspectos más tradicionales para el trabajo de una renovación curricular, sin embargo, se debe mencionar también aspectos que hoy en díase deben incorporar en esta discusión, a saber:

1. La revisión curricular hoy en día debe considerar dos conceptos, primero Co-producción (Coproduction), modelo que se orienta a un factor clave, la asociatividad con actores claves para forma- 
ción de odontólogos (Ministerio de Salud, Departamentos de Salud Municipal, Convenios con organizaciones de salud, etc.), cuyas coaliciones estratégicas a largo plazo logran impactar formativamente en el profesional que se está formando en el área odontológica; sobre todo en la etapa final de la formación, la Práctica Profesional (Isovic et al., 2013). $Y$ segundo, el Diseño de pensamiento (Designthinking), herramienta que logra soluciones específicas en base a una variedad de soluciones $u$ opciones de mejora ante los desafíos o problemáticas profesionales que se presenten en el trabajo (Urroz-Osés, 2018). Esta herramienta, no aplica solo a equipos de trabajo, sino que también sirve de sobre manera para aquellas profesiones de corte más individual de desempeño profesional -como es el caso de la odontología - pues la lógica de trabajo y el espacio donde se desenvuelve el quehacer profesional odontológico requiere de una alta exigencia para la optimización de los resultados esperados de los procedimientos, pero también su equipo de apoyo debe estar sintonizado a esta lógica laboral para que en conjunto resuelvan de mejor manera los desafíos que depara el ejercicio de la odontología.

2. Incorporar a las ciencias sociales con la finalidad de que éstos saberes o herramientas desde las sociales para la formación profesional procedimental de un odontólogo puedan ser aplicadas con mayor y mejor pertinencia socio-cultural donde se desarrolla el ejercicio profesional (Payares, 2007), ya que las ciencias sociales entregan:

-Conocimientos y saberes de cómo se dan las interacciones entre los individuos y la sociedad, ya que la interacción entre el profesional y el paciente involucra no tan solo a ellos dos actores, sino que también están los contextos sociales de ambos, y esa interrelación que se da en la atención de pacientes, hace que sea necesario una formación profesional que involucre estos saberes sociales para que tengan mejores habilidades para desenvolverse en el futuro como titulados.

- Aprendizajes de destrezas comunicacionales entre estudiantes, entre estudiante y docentes, estudiantes y funcionarios clínicos, y especialmente estudiantes y pacientes para ejercitar la interacción y generación de lazos con sus futuros pacientes para el logro de tratamientos eficientes y eficaces en su atención.

3. La búsqueda de la sustentabilidad y el cuidado del medio ambiente en la utilización de elementos pro- pios de la formación odontológica y de su ejercicio profesional.

4. Identificar las patológicas y sus tratamientos futuros de acuerdo a la legislación vigente el odontólogo generalatenderá en las comunidades donde se desenvolverá.

5. Como unos adecuados hábitos en salud son relevantes en la formación para estudiantes son relevantes en su proceso formativo, referentes a:

- La alimentación.

- El ejercicio diario.

- La higiene de sueño.

6.Como adquirir las herramientas para ser más resilentes para enfrentar las críticas, fracasos y evaluaciones negativas durante su formación profesional.

7.La prevención de enfermedades mentales y musculoesqueléticas que tienen un mayor desarrollo en las carreras de la salud.

8. Un preclínico dinámico e interactivo para el estudiante que eventualmente lo pueda complementar durante su formación clínica.

9. Revisar las actividades teóricas no tan solo sean tradicionalmente como entrega de material sino que más bien incorporen técnicas interactivas de enseñanza.

10. El manejo y comprensión de los alcances que tiene los conceptos de ética, moral, bioética, legislación y políticaspúblicas en el ámbito de la odontología.

11. La búsqueda del justo equilibrio en la incorporación de tecnologías para el diagnóstico y tratamiento en el ámbito odontológico en la formación del pregrado.

12. La capacidad de adecuación y optimización de los protocolos clínicos en base a una segura bioseguridad, esto cobra especial relevancia a propósito del COVID-19.

\section{CONCLUSIONES}

En definitiva, la revisión curricular para la carrera de Odontología de la Universidad de La Frontera que está planteada es de carácter abierta, modificable y adaptable -ya que no está orientada a priori a ningún modelo o metodología de enseñanza en aula - porque además es relevante destacar que una vez iniciado el trabajo se deberá cuidar el equilibrio entre lo metodológico y lo profesional. De esta forma se evite que todos los esfuerzos estén en el manejo y dominio en lo metodológico del modelo curricular seleccio- 
nado para este proceso y sea finalmente que éste quede por sobre lo profesional específico de la formación del cirujano dentista general; ya que el fin de la renovación curricular es que se logren las competencias profesionales específicas que tributan y caracterizan el perfil de egreso de un cirujano dentista que el país requerirá en el futuro y que en el proceso formativo le de la atmosfera adecuada a todos los actores para que siempre el centro sea la salud de las personas.

FUENTES, F. R. \& RIQUELME, M. C. Background and considerationsforthe curricular renewal of the Universidad de La Frontera, Chile dental degree. Int. J. Odontostomat., 15(1):234-239, 2021.

ABSTRACT: The objective of this article is to contribute to the discussion and reflection of the theoreticalconceptual elements that should be incorporated in a process of curricular renewal. In this case, concerning the dental degree of the Universidad de La Frontera, Chile, this process should be geared toward the new work context required in the dental field nowadays. Clear designs and guidelines for the development of students' meaningful learning should be established. These should include Interdisciplinary knowledge, technical competences and specific educational tools in a comprehensive educational model for the students and subsequent professionals. Faculty as well as students ought to be included in all stages of this process. To accomplish this challenge in education and training requires a significant and absolute commitment on the part of all players involved, given that the objective is the substantial improvement of the dental professional education in the La Araucanía region.

\section{KEY WORDS: curriculum renewal, teaching- learning process, professional skills.}

\section{REFERENCIAS BIBLIOGRÁFICAS}

Améstica-Rivas, L.; Llinas-Audet, X. \& Escardíbul, J. Costos de la Renovación Curricular. Una Propuesta Metodológica. Form. Univ., 10(1):89-100, 2017.

Daza J. Renovación curricular en programas de ciencias de la salud y su impacto en las prácticas pedagógicas de los profesores. Rev. Cienc. Salud, 8(1):71-85, 2010.

Díaz-Barriga, F. Los profesores ante las innovaciones curriculares. Rev. Iberoam. de Educ. Super., 1(1):37-57, 2010.

Duarte, C. \& Mora, A. Renovación curricular, una oportunidad para repensar el Trabajo Social en la Universidad de Atacama. Rev. Docencia Univ., 14(1):203-23, 2016.

Fuentes, F. R.; Henriquez, P. J.; Matamala, V. F. \& Sandoval, V. P. Curriculum innovado de la Carrera de Odontología, Facultad de Medicina. Odontol. Chil., 44(1):35-9, 1996.
Fuentes, R. \& Mardones, D. Bases académicas del proyecto Carrera de Odontología de la Universidad de La Frontera. Odontol. Chil., 41(1):67-70, 1993.

Isovic, D.; Wallin, F. \& Gustafsson, C. The coproductive university. Education and research in coproduction with the wider community. Conferencia. University Industry Integration Conference, UIIC, 2013.

Matamala, F.; Cabalin, D.; Thomas, D.; Pantoja, M.; Fuentes, R. \& Mardones, D. Perspectivas de las Ciencias Morfológicas en el Currículo innovado de la Carrera de Odontología, Universidad de La Frontera. Rev. Chil. Anat., 10(2):121-6, 1992.

Miranda, K. Formación académica en odontología ante los retos del mercado laboral y los entes reguladores de aprobación y acreditación. Revista electrónica de la Facultad de Odontología, ULACIT (Costa Rica), 6(1):2-16, 2013.

Pantoja, M. A.; Cabalin, D.; Matamala, F.; Mardones, D. \& Fuentes, R. Creación de la Carrera de Odontología, Universidad de La Frontera, Temuco: un curriculum innovado. Rev. Chil. Docencia Serv. Investig. Salud, 1993.

Payares, C. La incorporación de las ciencias sociales en la formación del recurso humano odontológico. Duazary, 4(2):168-74, 2007.

Pey, R. \& Chauriye, S. Innovación Curricular en las Universidades del Consejo de Rectores 2000 - 2010. Santiago de Chile, Consejo de Rectores de las Universidades Chilenas (CRUCH), 2011.

Ríos Muñoz, D. \& Herrera Araya, D. Los desafíos de la evaluación por competencias en el ámbito educativo. Educ. Pesqui. (São Paulo), 43(4):1073-86, 2017.

Ruay, R.; González, P. \& Plaza, E. ¿Cómo abordar la renovación curricular en la educación superior?, Alteridad, 11(2):157-70, 2016.

Urroz-Osés, A. Diseño y desarrollo: la innovación responsable mediante el Design Thinking. Cuad. Cent. Estud. Diseño Comun., 19(69):195-206, 2018.

Vicerrectoría de Pregrado (VIPRE). Manual de Diseño Curricular. Temuco, Vicerrectoría de Pregrado, Universidad de La Frontera, 2018.

Vicerrectoría de Pregrado (VIPRE). Política de Formación Profesional. Temuco, Vicerrectoría de Pregrado, Universidad de La Frontera, 2008.

Dirección de correspondencia:

Ramón Fuentes

Facultad de Odontología

Universidad de la Frontera

Temuco

CHILE

Email: ramon.fuentes@gmail.com 